DOI: https://doi.org/10.34069/RA/2021.8.05

Volumen 4, Número 8/julio-diciembre 2021

Reyes Galardy, Y., \& Estrada Silveira, J. (2021). Evolución histórica del desarrollo de habilidades infotecnológicas en la educación primaria. Revista Científica Del Amazonas, 4(8), 49-58. https://doi.org/10.34069/RA/2021.8.05

\title{
Evolución histórica del desarrollo de habilidades infotecnológicas en la educación primaria
}

\section{Historical evolution of the development of infotechnology skills in primary education}

\author{
Recibido: 26 de abril de $2021 \quad$ Aceptado: 2 de junio de 2021
}

\author{
Autores: \\ Yoesky Reyes Galardy ${ }^{20}$ \\ Jorge Estrada Silveira ${ }^{21}$
}

\section{Resumen}

Este artículo hace referencia a la evolución histórica del desarrollo de habilidades infotecnológicas en el segundo ciclo de la Educación Primaria. En tal sentido, se define para su estudio, el período comprendido desde el año 2001 hasta la actualidad que comprende la introducción masiva de la computación en la Educación Primaria, con dos etapas, donde se evidencia el perfeccionamiento continuo que experimenta el Sistema Educacional Cubano en el proceso de enseñanza-aprendizaje de la computación y los esfuerzos que ha realizado el país por poner la educación al nivel del desarrollo tecnológico logrado a nivel global. Para el análisis de cada una de estas etapas, se tienen en cuenta indicadores que permiten la profundización en las transformaciones aplicadas, la preparación científico-metodológica de los maestros de informática, características del programa de informática en el segundo ciclo de la educación primaria, métodos de búsqueda de la información y herramientas infotecnológicas utilizadas. Este análisis revela las principales características de las etapas y las tendencias generales del estudio histórico realizado a partir de los indicadores seleccionados.

Palabras clave: aprendizaje, computación, enseñanza, habilidades, herramientas infotecnológicas.

\begin{abstract}
This article refers to the historical evolution of the development of infotechnology skills in the second cycle of Primary Education. In this sense, it is defined for its study, the period from 2001 to the present time that includes the massive introduction of computers in Primary Education, with two stages, where it is evidenced the continuous improvement that the Cuban Educacional System is experiencing in the teaching-learning process of computers and the efforts that the country has made to put education at the level of the technological development achieved at a global level. For the analysis of each one of these stages, indicators are taken into account that allow the deepening of applied transformations, the scientific-methodological preparation of computer science teachers, characteristics of the computer science program in the second cycle of primary education, methods of information search and infotechnological tools used. This analysis reveals the main characteristics of the stages and general trends of the historical study carried out on the basis of the selected indicators.
\end{abstract}

Key Words: learning, computing, teaching, skills, infotechnology tools.

${ }^{20}$ Licenciada, Universidad de Granma, profesora, Granma, Cuba, https://orcid.org/0000-0003-4314-9625

${ }^{21}$ Ingeniero, Delegación Provincial CITMA, Especialista, Granma, Cuba, https://orcid.org/0000-0002-6125-4376 


\section{AMAZONANAS}

\section{Introducción}

El sistema educacional en Cuba está inmerso en un proceso de transformaciones con el objetivo de elevar la cultura general integral de las nuevas generaciones, para ello la escuela debe ser un espacio movilizador de la capacidad intelectual, la creatividad y del sentido innovador de los conocimientos generados, acorde con el medio social en el que se haya insertada.

Es por ello que en los últimos años se ha potenciado la introducción de la computación en la escuela Primaria para que el escolar en toda su actividad tenga un papel activo, crítico, reflexivo, independiente, protagónico en su actuación, a través de un proceso de enseñanzaaprendizaje participativo, flexible, regulado, donde las tecnologías informáticas tienen un importante rol.

En la Educación Primaria, la asignatura Computación cuenta con un programa básico, en el que el profesor, para lograr los objetivos, contenidos y habilidades que se especifican por grados, debe aprovechar las potencialidades que brindan las herramientas infotecnológicas a su alcance, en función de facilitar la asimilación y comprensión del contenido por parte de los escolares.

De ahí que, el uso de las herramientas infotecnológicas sirva para que el escolar actúe de manera independiente en la búsqueda de sus propios conocimientos y los apliquen en contextos diversos de su esfera de actuación, en consonancia con la creciente interrelación de la informática con las diferentes asignaturas.

La búsqueda de información utilizando la Infotecnología permite activar los procesos lógicos del pensamiento y propicia la argumentación sobre la concepción científica acerca de la naturaleza y la sociedad, favoreciendo la apropiación de los conocimientos con un enfoque integral en la solución de problemas de la práctica social.

Una de las causas fundamentales de esta situación resultó ser el insuficiente e ineficaz enfoque didáctico del empleo de las herramientas infotecnológicas en el proceso de enseñanzaaprendizaje. En tal sentido, las herramientas infotecnológicas integradas con los medios y métodos de enseñanza y aprendizaje de las asignaturas propician el marco teórico para favorecer la apropiación de conocimientos y su aplicación a la solución de problemas, en los escolares del segundo ciclo de la escuela primaria.

La presente investigación se ha nutrido de los trabajos realizados por diversos autores que han afrontado el estudio de la Infotecnología, entre ellos se encuentran: Ramírez (2011), trata el desarrollo de las habilidades en la Web así como la creación de bibliotecas digitales para los estudiantes; Torricella (2012), plantea la creación de bibliotecas digitales para las tesinas y así sirvan como herramientas para la capacitación de los profesores y estudiantes; González (2013) quien asume la creación de bibliotecas digitales

Por otra parte, Ramírez (2011), asume la gestión de información; Suárez (2015), para el desarrollo de habilidades en la Web en los estudiantes universitarios; Prieto (2021), la asume como: estrategia de trabajo de sofisticadas herramientas, organización y procesamiento de la información digital.

Tanto los profesores como los escolares necesitan de conocimientos sobre las habilidades infotecnológicas en el proceso de enseñanza-aprendizaje. Es insuficiente la metodología que se le brinda al profesor con una lógica a seguir para desarrollar habilidades infotecnológicas en los escolares del segundo ciclo de la escuela primaria, así como vías para que el escolar ejercite y pueda disfrutar de las habilidades adquiridas que le puedan servir para su vida diaria. 
Desde el punto de vista didáctico constituye un reto el desarrollo de las habilidades infotecnológicas en los escolares del segundo ciclo de la escuela primaria, en tanto actualmente están dirigidas específicamente a la búsqueda de información y navegación de la Web en los escolares primarios.

En las perspectivas y enfoques de los investigadores que han estudiado la Infotecnología, aún no se aborda el desarrollo de las habilidades infotecnológicas en los escolares del segundo ciclo en la Educación Primaria, siendo una necesidad fundamental en el proceso de enseñanzaaprendizaje de la Computación donde actualmente existen insuficiencias.

Por tales razones, en el desarrollo del artículo se revelan las características fundamentales y tendencias generales en la evolución histórica del desarrollo de habilidades infotecnológicas en el segundo ciclo de la Educación Primaria, las que demuestran la existencia de insuficiencias que necesitan ser solucionadas por la vía investigativa.

\section{Materiales y métodos}

La investigación tuvo carácter explicativo y siguió el enfoque interpretativo. Fue desarrollada en cuatros centros de la provincia Granma pertenecientes a la Educación Primaria. Además, se trabajó con 30 profesores representantes de esta enseñanza. En el tiempo de la investigación fueron incluidos en la población 15 profesores con más de 20 años de experiencia impartiendo la asignatura Computación.

Los métodos esenciales utilizados fueron el análisis de documentos, la entrevista, la prueba pedagógica sobre el objeto estudiado. Aunque la mayor información se obtuvo a partir del período revolucionario se consideró necesario comenzar con un breve análisis sobre lo sucedido desde el siglo XIX en relación con la temática estudiada.

\section{Resultadosy discución}

Uno de los primeros resultados obtenidos de la aplicación de los instrumentos en relación con el desarrollo de las habilidades infotecnológicas, evidenció que entre los antecedentes de la introducción de la Computación de forma masiva en la escuela primaria se encuentran los siguientes:

En el año 1987 se inauguró en el Palacio Central de Pioneros "Ernesto Guevara" un círculo de interés de computación electrónica, donde los niños disponían de una CID 300/10, teclados inteligentes con el lenguaje BASIC incorporado, conectados a televisores y a grabadoras de casetes, una microcomputadora profesional IBM compatible, calculadoras y algunos equipos electrónicos para prácticas. Este círculo se dirigía a niños de edades tempranas, y fue el germen de lo que se llamarían después "Joven Club de Computación".

En el curso 1986-1987 comienza la preparación de los maestros en las técnicas de computación, al estudiarse en las escuelas Formadoras de Maestros y se procedió a la instalación de computadoras en 120 escuelas primarias, con carácter experimental, en las que se imparte el lenguaje de programación en MSX-LOGO en segundo ciclo y se utilizaron software educativos fundamentalmente en primer grado con el propósito de contribuir al desarrollo intelectual de los escolares, a la asimilación de conocimientos de diferentes asignaturas y ofrecer una cultura informática elemental como parte de su formación integral.

Posteriormente se extendieron las experiencias a 157 escuelas primarias del país, donde se concibió el turno de Computación como una actividad complementaria de apoyo a la docencia. A pesar de la novedad que constituía el uso de la computación por primera vez en el nivel primario 


\section{AMAZÉNAS}

y de la motivación, este proyecto se sustituyó por el estudio del lenguaje MSX-BASIC, residente en tableros electrónicos de 8 bit, que resultaban más económicos.

En septiembre de 1987 se crearon los Joven Club de Computación y Electrónica, proyecto social de la Unión de Jóvenes Comunistas al que asisten niños, adolescentes, jóvenes y todas las personas que quieren acercarse al mundo de la computación a través de actividades instructivas, vocacionales y recreativas. Con este paso se contribuyó a la introducción de la Computación como asignatura en la Educación Primaria.

En 1988 se comienza la realización de una experiencia en el primer grado de la enseñanza primaria mediante juegos instructivos y en quinto y segundo ciclo grados con elementos de programación.

El criterio de periodización asumido es: los cambios fundamentales operados en los programas de Computación y su papel, en el proceso de enseñanza-aprendizaje en la Educación Primaria, por lo que para realizar una caracterización del objeto de investigación se consideraron, fundamentalmente, los siguientes hitos históricos:

- En el año 2001 se introduce de manera masiva en todas las escuelas primarias del país la enseñanza de la Computación.

- En el año 2012 se introducen cambios sustanciales en el Programa de Computación en la Educación Primaria.

Para guiar el análisis tendencial del objeto de investigación se consideraron, fundamentalmente, los siguientes indicadores:

Preparación científica y metodológica de los profesores de informática. Características del programa de informática en el segundo ciclo de la Escuela Primaria; Métodos de búsqueda de la información; Herramientas infotecnológicas utilizadas.

Para analizar cómo ha transcurrido la enseñanza de la asignatura Computación en el segundo ciclo de la educación primaria, se utiliza el método histórico-lógico a través de dos etapas:

Primera Etapa: (2001-2011): introducción de la Computación en la educación primaria.

Segunda Etapa: (2012 hasta la actualidad): perfeccionamiento del proceso de enseñanzaaprendizaje de la Computación en la educación primaria.

Las características del objeto de la investigación, en cada una de las etapas y períodos, se analizan a continuación:

\section{Etapa desde 2001 hasta 2011.Introducción de la Computación en la Educación Primaria.}

El objetivo fundamental de la computación en la Educación Primaria en sus inicios fue para aumentar la calidad del proceso de enseñanza-aprendizaje y buscar asegurar el futuro del país, Díaz manifiesta: que “... no se pretende esencialmente enseñar Informática como asignatura, lo cual está incluido, sino utilizarla para mejorar y potenciar la enseñanza de todas las asignaturas y educar a los niños y jóvenes". (2006, p. 48). Además, la preparación de las nuevas generaciones en la utilización de las tecnologías y el empleo de estos recursos permite un mayor desarrollo en el aprendizaje en los escolares. 
Para poder introducir la Computación en las escuelas primarias del país, fue necesario preparar, de forma emergente, a un grupo de maestros para impartir la asignatura, y desarrollar un curso básico inicial de Computación para todos los maestros en el que se les preparaba mínimamente para operar la computadora y utilizarla como medio de enseñanza en la dirección del proceso de enseñanza-aprendizaje.

Se propone un Programa de Informática con el objetivo de capacitar a los maestros primarios para integrar en sus clases los recursos y medios informáticos, fue concebido en dos cursos de 32 y 44 horas respectivamente. La concepción de los programas garantizaba los contenidos básicos para continuar la formación informática de todos los maestros primarios, sin embargo, la sensibilización y motivación de maestros y directivos hacia su empleo no fue completamente logrado en consonancia con la necesidad de utilizar los avances tecnológicos en el proceso de enseñanza-aprendizaje.

En esta etapa se comienza a estudiar la Computación como objeto de estudio, como aplicación y como medio de enseñanza en todas las escuelas primarias, así como a utilizar diferentes tipos de softwares educativos que para la enseñanza primaria se crean, entre ellos los software de la colección "Multisaber", que contiene 32 software (31 de primaria y uno de preescolar) destinados a contribuir al desarrollo del proceso de enseñanza-aprendizaje, los cuales tienen un carácter curricular, lo que favorece su aplicación en las distintas asignaturas, se comienza a utilizar las teleclases y los videos como medio de enseñanza para impartir los contenidos de las asignaturas, las que además servían para la auto preparación de los maestros. Al utilizarlos como consolidación de contenidos, se descuidó la preparación de los escolares del segundo ciclo en el uso de estos para el desarrollo de las habilidades informáticas y el cumplimiento de los objetivos del modelo de este nivel de educación.

En el proceso de enseñanza-aprendizaje del segundo ciclo de la Educación Primaria y con ella el uso de software educativos teniendo en cuenta sus potencialidades metodológicas, permiten que los escolares interactúen de forma independiente y dirigida con los contenidos, que desarrollen estrategias de aprendizaje, reciban la ayuda que aparece en los software, hagan búsqueda de información, interactúen con representaciones de procesos naturales en movimiento que en otras condiciones es muy difícil y logren mejores resultados en el proceso de enseñanza aprendizaje.

Las tecnologías son utilizadas como medio de enseñanza para resolver las insuficiencias que en el desarrollo de las habilidades informáticas presentan los escolares, lo que evidencia la necesidad de utilizar nuevas herramientas y medios de enseñanza que se correspondan con la nueva era tecnológica que vive la educación. Estas habilidades a desarrollar en la Educación Primaria a partir del uso de los softwares educativos de la Colección Multisaber son:

Identificar las características generales de la computadora (partes y funciones elementales), utilizar el procedimiento para encender la computadora, realizar acciones con el ratón (clic, doble clic y arrastre), reconocer los íconos y accesos directos de los softwares educativos en el escritorio de Windows y en el menú de Inicio. Localizar y ejecutar las aplicaciones, interactuar con los softwares educativos que se recomiendan para este grado, realizar y modificar dibujos sencillos utilizando un editor gráfico, utilizar el teclado (escribir), ejecutar los procedimientos de guardar y abrir archivos (dibujos y textos) elaborados, identificar elementos de organización de la información en los dispositivos carpetas y archivos, realizar el control y la valoración de los resultados de sus trabajos y de sus compañeros a partir de indicadores dados por el maestro e incorporarlos a sus acciones.

Además de selección y extracción de la información hacia otras aplicaciones informáticas, búsqueda y uso de la información: botón buscar, resolver problemas prácticos 


\section{AMAZONANAS}

relacionados con las asignaturas del grado escolar que cursan, al utilizar la computadora como herramienta y medio de enseñanza para la búsqueda y utilización de información, hipertextos (palabras calientes), libro electrónico e hipervínculos.

La utilización de software permitía elevar el conocimiento de los escolares siendo insuficiente las habilidades infotecnológicas adquiridas a la hora de buscar información que les permitiera elevar el aprendizaje.

En las escuelas primarias del país se crearon todas las condiciones para instalar los laboratorios de computación, para ello fue necesario electrificar con paneles solares 2368 escuelas. Todas estas medidas se concibieron para lograr mejores resultados en los objetivos del proceso de enseñanza-aprendizaje y contribuir a la formación integral de la personalidad de los escolares, se instalaron 24000 computadoras modernas en la totalidad de las escuelas (8 883), entre las que se incluyeron 99 escuelas con matrícula de un solo niño.

Es ese mismo año se implementó la interacción con las enciclopedias electrónicas, los escolares lo hacían como un entretenimiento y para el cumplimiento de tareas orientadas que propiciaran la búsqueda de información y así enriquecer el proceso de enseñanza-aprendizaje.

Se implementaron dos programas de estudio, uno destinado a los escolares de primer a tercer grado y el otro para los de cuarto a segundo ciclo, con el objetivo de preparar a los escolares en las habilidades informáticas para dar solución a tareas relacionadas con los contenidos de las diferentes asignaturas, lo que limitó el proceso de enseñanza-aprendizaje.

Posteriormente se puso en práctica otra propuesta de programa de Computación en el mismo curso escolar, que estaba mejor concebida que la primera, pero con mucho contenido para los escolares del segundo ciclo de la Educación Primaria. Para el curso 2002-2003 se les facilitó a los maestros un programa de Computación con sus orientaciones metodológicas, en las que se incluían recomendaciones para el uso del software educativo, la solución de tareas sencillas para apropiarse del contenido y hacer más ameno el proceso de enseñanza aprendizaje. (Ulloa, L. 2006, p.37).

En esta etapa a los maestros se le dificulta utilizar la Computación es sus clases, debido a la falta de preparación y a la disponibilidad de recursos. Algunos consideraban las tecnologías como una carga más y no como una herramienta para lograr mejores resultados en los objetivos al establecer relaciones con el contenido y motivación de las clases. Las actividades no se desarrollaban en toda su plenitud para utilizar las potencialidades de las tecnologías y se continuaba con un proceso de enseñanza-aprendizaje con tendencias tradicionalistas que no promueve suficientemente el desarrollo aspirado.

De forma paulatina a cambiar la tecnología de las computadoras en su mayoría por Pentium V con 1 GB de RAM y 500 GB de capacidad de disco duro, con lo que se mejora el rendimiento, la velocidad y la capacidad de almacenamiento. Este aspecto permite aprovechar las bondades de las Tecnologías de la Información y las Comunicaciones (TIC) para elevar la calidad del proceso de enseñanza aprendizaje y lograr los objetivos a un mayor nivel, sin embargo, fue insuficiente el uso de las TIC para que los escolares del segundo ciclo buscaran información, la procesaran y la emplearan para resolver tareas tanto docentes como de la vida.

En esta etapa ocurrieron cambios importantes en los programas de estudio de Computación, los softwares educativos se implementaron en el currículo para consolidar y ejercitar los contenidos de las asignaturas, se utilizaron de manera formal, lo que limitó su empleo en la búsqueda activa de información, procesamiento, elaboración y comunicación de nuevos 
conocimientos que contribuyeran al desarrollo del proceso de enseñanza aprendizaje aspirado en las exigencias sociales.

Cuando en las escuelas primarias se instalaron las enciclopedias, se mantuvieron los softwares de la Colección Multisaber, y se comienza a implementar las clases y las tareas con software educativos o softareas, con el objetivo de apoyar el proceso de enseñanza-aprendizaje, que unido al tiempo de máquina de los escolares ocupó uno de los espacios fundamentales para desarrollar las habilidades infotecnológicas, aunque no se aprovecharon estas potencialidades en toda su magnitud.

Se instala en las escuelas primarias la Enciclopedia Colaborativa cubana Ecured portable, así como el acceso a Internet o Intranet y por ende la visualización de algunas páginas como Cubadebate, las listas de discusión, el Chat, la WEB, entre otras páginas importantes nacionales que promueven la búsqueda, selección, procesamiento y desarrollo de habilidades informáticas, lo que permitió el desarrollo de las potencialidades de los escolares del segundo ciclo.

Al igual se distribuyeron las horas clases y se agregó el tiempo de máquina a segundo ciclo, se propició una mayor interacción con los softwares educativos para apoyar el contenido a tratar en las asignaturas, con el objetivo de obtener mejores resultados en el proceso de enseñanza aprendizaje. Aunque se crearon mayores espacios para la preparación e interacción con estos medios fue insuficiente su intencionalidad didáctica para el desarrollo de las habilidades y el uso de herramientas infotecnológicas. Del mismo modo que aunque se dieron pasos en la utilización de los software, las softareas, las herramientas infotecnológicas, no se logró un adecuado trabajo metodológico en tal sentido.

En las escuelas primarias se utiliza la computadora en el recibimiento y despedida de los escolares para que realicen tareas, trabajos prácticos y extraclase, aún insuficientes en el proceso de enseñanza-aprendizaje, en la recreación sana y en la relación de la computadora con las demás asignaturas, sin embargo, teniendo la tecnología en sus manos la búsqueda de información es poca a través de las herramientas existentes en la computadora.

La utilización de los softwares educativos es una de las vías que tiene el estudiante bajo la orientación del maestro para la búsqueda automática, interactuar con otros escolares, apropiarse de información necesaria y para coordinar actividades que le permitan desarrollar habilidades informáticas.

En las escuelas urbanas de la Educación Primarias, la Intranet es sólo para los escolares que tienen familiares cumpliendo misión en otros países y para que se puedan comunicar se les abrió una cuenta internacional. Para los demás escolares y maestros tenían la conexión a Intranet para la búsqueda de información en los centros que tienen conexión y posibilidades que brinda la escuela para adquirir habilidades informáticas.

\section{Etapa desde 2012 hasta la actualidad. Perfeccionamiento del proceso de enseñanza- aprendizaje de la Computación en la Educación Primaria.}

En esta etapa se comienza a distribuir un programa que se imparte en quinto grado: consolidando lo aprendido de grados anteriores, además, en segundo ciclo, se imparten los mismos contenidos insertando una unidad: demostrando lo aprendido, en el cual no aparece ningún tema relacionado con la Intranet ya existiendo en las escuelas primarias desde el 2005, además no es utilizado en tiempos de máquina ni en la recreación sana.

Con la introducción de la Computación se asumen cambios en la organización del proceso enseñanza-aprendizaje desde la concepción curricular, centrados en un modelo más humanista, 


\section{AMAZÉNAS}

heurístico, flexible y desarrollador que favorece transformaciones en las maneras de pensar, sentir y actuar.

En estas adecuaciones no se incluyó el trabajo con la intranet, en las escuelas con disponibilidad técnica. Las Tecnologías de la Información y de la Comunicación han evolucionado espectacularmente en los últimos años, debido especialmente a su capacidad de interconexión a través de la Red. La acomodación del entorno educativo a este nuevo potencial y la adecuada utilización didáctica del mismo, lo cual supone un reto sin precedentes.

En esta etapa la utilización del programa de Computación brinda varias horas clase para la consolidación y evaluación de los contenidos. Se orientaron trabajos prácticos por los maestros en las diferentes asignaturas; desarrollando habilidades informáticas esencialmente en la búsqueda de información que aún es insuficiente. Además, el trabajo con la Ecured y Wikipedia fue importante en esta etapa, los escolares buscan información orientada por los maestros y alcanzan habilidades informáticas.

Con la introducción del correo electrónico se propició una habilidad informática fundamental en la era moderna por la necesidad continua de comunicarse con personas de todo el mundo, esto les permitía comunicarse con sus familiares, sus compañeros de grado y maestros de otras escuelas posibilitando un mayor flujo de información y con ello un avance cualitativo y cuantitativo en el desarrollo de habilidades infotecnológicas en los escolares del segundo ciclo.

Se demostró como aspecto efectivo que se emplearon los avances científicos y tecnológicos para un mejor desarrollo del proceso de enseñanza-aprendizaje. Además, se estableció el flujo de comunicación mediante la Intranet, Internet, correos electrónicos, Ecured y otras tecnologías que propician el desarrollo, así como la aplicación de las tecnologías para la elaboración de trabajos prácticos e independientes y extraclases.

Se reconocieron las potencialidades del uso de los diccionarios digitales, enciclopedias como: Ecured y Wikipedia, y acceso a sitios Web para obtener información. A pesar de ello, es insuficiente el desarrollo de habilidades informáticas en el segundo ciclo en el proceso de enseñanza-aprendizaje. Se favoreció la utilización de software educativo que permiten profundizar en los contenidos orientados en clases, se estableció el flujo de comunicación mediante las tecnologías que propician el desarrollo y la aplicación de las mismas para la elaboración de trabajos prácticos e independientes. No obstante, por la falta de una metodología que permitiera concretar el desarrollo de habilidades infotecnológicas, no se logró la concreción necesaria en la práctica por parte de los escolares del segundo ciclo de la Educación Primaria.

Desde los años 2010 hasta el 2014 se crearon nuevos software para el estudio de la historia como: Mausoleo 11 Frente Oriental; Memorial Granma; Camilo aquí está el Che; Casa museo de Abel Santamaría; Visita virtual complejo escultórico Cacahual y Memorias de la Guerra para el estudio de la Historia de Cuba que permitieron obtener información en la ejecución de actividades para desarrollar habilidades informáticas en los escolares, así como utilizar herramientas como navegadores, buscadores entre otras y elevar los resultados en el cumplimiento de los objetivos.

Además se introdujo la enseñanza de la Computación como una asignatura complementaria; se hizo llegar a cada escuela los Programas y Orientaciones metodológicas de la misma para contribuir a la formación integral de la personalidad de los escolares; se contempla el uso de las TIC en la solución de tareas, elaboración de trabajos prácticos e independientes que evidencian un paso positivo en los medios tecnológicos, aunque no se logró introducir acciones que permiten el uso de la Intranet para el desarrollo de las habilidades infotecnológicas en el segundo ciclo de la Educación Primaria. 
Se distribuyó por primera vez a todas las escuelas primarias un programa para la asignatura Computación en soporte plano el cual contiene las Orientaciones Metodológicas. En el programa de Computación se dedicaron varias horas clases para la consolidación y evaluación del contenido. Se orientaron trabajos prácticos sobre temáticas determinadas por los maestros; fundamentalmente en la búsqueda de información. Según el contenido a tratar el maestro con un navegador o la guía de software educativo busca el tema que le interesa para que los escolares interactúen, se apropien de la información que necesitan y coordinen actividades para realizar tareas o investigar algún tema de interés, además les orienta que busquen información utilizando alguna de las herramientas infotecnológicas y que tomen notas que les permitan a los escolares desarrollar habilidades informáticas.

En esta etapa fue fundamental la conexión a Intranet, durante el tiempo de máquina, para la búsqueda de información necesaria para el desarrollo cultural de los estudiantes, en aquellas escuelas con conexión. Además, se potenció la utilización de la enciclopedia Ecured para la búsqueda de información orientada por los maestros y las comparen con la que aparece en los libros de textos y otros materiales de consulta para llegar a juicios particulares.

La computadora es un medio de enseñanza de gran utilidad para las tareas asignadas a los escolares, pero se descuidan sus motivaciones y potencialidades de aprendizaje; el tiempo de máquina centró su atención a tareas formales, reproductivas que no potencian la independencia y el desarrollo para buscar información, procesarla, emplearla y comunicarla en trabajos extraclases, prácticos e independientes. Se reconocieron las potencialidades del uso de los diccionarios digitales, Ecured, Wikipedia, sitios Web para obtener información.

La Internet e Intranet son utilizadas insuficientemente por los maestros y escolares, no se le da tratamiento a la búsqueda de información en algunos navegadores, se desconoce el uso del correo electrónico entre otras herramientas que tienen en sus manos. Desconocimiento de las herramientas infotecnológicas como: navegadores Web (Mozilla Firefox, Opera, Internet Explorer, la Web 2.0 (wiki, blog, redes sociales), buscadores (Google, Yahoo, Bin. Twiter) y correo electrónico (e-mail).

Del análisis histórico realizado se pudo determinar los elementos fundamentales del diagnóstico del proceso de enseñanza-aprendizaje de la asignatura Computación en el segundo ciclo y el desarrollo de las habilidades infotecnológicas en esta enseñanza, lo que le permitió comprenderlo en su historia, en su desarrollo y lógica; y por tanto precisar la necesidad de dar respuesta a las insuficiencias detectadas por medio de la investigación científica.

\section{Conclusiones}

Del análisis histórico realizado se pudo determinar las características de los elementos fundamentales del diagnóstico del proceso de enseñanza aprendizaje de la asignatura Computación en el segundo ciclo de la Educación Primaria, referidas a que, la preparación tanto científica como metodológica de los profesores en esta enseñanza aún están con el aprendizaje tradicional sin darle un mayor uso al desarrollo de las habilidades infotecnológicas, la tecnología, la búsqueda de información en algunos navegadores, uso del correo electrónico y las nuevas herramientas infotecnológicas novedosas para la motivación y utilización gradualmente a los diferentes momentos del proceso en el logro de los objetivos.

El análisis y estudio de la teoría existente en relación con el proceso de enseñanza aprendizaje de la informática en la Educación Primeria permite determinar los referentes teóricos necesarios en el orden filosófico, sociológico, psicológico, didáctico y tecnológico. 


\section{AMAZÉNAS}

El estudio actual del proceso de enseñanza aprendizaje de la informática en la Educación Primaria permite comprobar la existencia de insuficiencias en el desarrollo de las habilidades infotecnológicas en los escolares primarios, siendo más significativas las relacionadas con el uso de las herramientas infotecnológicas.

\section{Bibliografía}

Díaz, G. (2006). Concepción teórico-metodológica para el uso de la computadora en el proceso de enseñanza-aprendizaje de la Educación Primaria (Tesis presentada en opción al grado científico de Doctor en Ciencias Pedagógicas), Instituto Superior Pedagógico "Enrique J. Varona, Ciudad de La Habana.

González, O, (2013). Escenarios 2020 para la biblioteca universitaria cubana: Tesis en opción al grado científico de Doctor en Ciencias Pedagógicas. Red de bibliotecas de la Universidad de La Habana. Cuba. (Material en soporte digital).

Prieto, O, (2021) Herramientas Multimedia Interactivas como Estrategia Pedagógica para Fortalecer Procesos de Enseñanza Aprendizaje en Docentes de la Básica Primaria. Bogotá, Colombia. https://repository.ean.edu.co/bitstream/handle/10882/10601/PrietoOscar2021.pdf?seque nce $=1 \&$ is Allowed $=\mathrm{y}$

Ramírez, R, (2011). La gestión de información y la Infotecnología en la formación del profesional de las Ciencias Económicas y Empresariales. Camagüey. Cuba. (Material en soporte digital).

Ramírez, R, (2011). La gestión de información y la Infotecnología en la formación del profesional de las Ciencias Económicas y Empresariales. (Material en soporte electrónico).

Suárez. (2015) La gestión de la información: herramienta esencial para el desarrollo de habilidades en la comunidad estudiantil universitaria. Revista Cielo. ISSN: 2218-3620. http://scielo.sld.cu/pdf/rus/v7n2/rus10215.pdf

Torricella, R, (2012). Perspectivas de la Infotecnología para la Editorial Universitaria (Cuba). Disponible en: http://eprints.rclis.org/14452/

Ulloa, L. (2006), Estrategia didáctica para la utilización de una colección de juegos por computadora en el primer grado de la Educación Primaria. (tesis doctoral) Instituto Superior Pedagógico José Martí, Camagüey, Cuba. 